\title{
Trend Analysis of the Costing of Denim Wash: A Study on Bangladeshi Washing Industries
}

\author{
Nazrima Sultana, Lecturer \\ Fareast International University, Bangladesh \\ Md. Arif Iqbal, Assistant Professor \\ Md. Abdus Salam, Research Scholar \\ Bangladesh University of Textiles, Bangladesh
}

Doi: 10.19044/esj.2019.v15n12p300 URL:http://dx.doi.org/10.19044/esj.2019.v15n12p300

\begin{abstract}
Denim industry is one of the largest in the global market due to its exotic fashion trend. Bangladesh is the largest exporter of denim products over the European market and just after China in worldwide production. Fashion sense in denim fabric is drastically increasing due to the introduction of multiple washing methods. It is a type of fabric finishing treated in a different way for the change of appearance. This paper focuses on the denim washing cost trend within the year 2010 to 2018, and it aims to find out various consequences to analyze the market status of denim washing. The study uses the primary data collected from ten leading denim washing factories in Bangladesh. The study shows that, around 2010, the trend of dry washing processes was not so familiar. Hence, around 2015, there was an extensive practice executed which dominates the conventional wet washing technologies. On the contrary, process like sandblasting got banned from around 2013 because of its harmful effects on workers and users. Besides, various expensive wet washing technologies like acid wash, stone enzyme wash, towel wash, brightener treatment, optical wash, etc. has been incorporated to provide quality washing effects. This paper shows the costing trend of different wash technologies. This is because the majority of the dry and wet wash technologies became more expensive with time gradually due to the increase in labor cost, utility costs, chemical, and process costs. Furthermore, it also shows that some new technologies, like laser technology, which provided effective cost with reasonable quality through the initial investments, are high. The paper gives a prediction for the upcoming trend of denim washing as well as the cost of the technologies.
\end{abstract}

Keywords: Denim, Washing, Costing, Trend Analysis, Bangladesh 


\section{Introduction}

Denim is a very strong, stiff, and hard wearing fabric. It is more than just a cotton fabric; it inspires strong opinions within the hearts of historians, designers, teenagers, movie stars, reporters, and writers. Interest bordering on passion can be found among textile and costume historians today, especially in the debate over the true origins of denim. Bangladesh is now one of the most important centers for denim apparel production and export across the world. It is now the largest exporter in the European Market and the second largest producer of denim products after China. With thousands of small and large garment units in Bangladesh, it is also one of the cheapest production centers (in and around the city of Dhaka and some around Chittagong) for apparel around the world. Since the commencement, worldwide market of denim remains evergreen. Centuries ago, denim had limited its usage as a work-wear, but with the versatility of its utility now, the demand for denim is always sky high in the global market. Irrespective of any market condition, denim gets ready acceptance to the consumers. Denim is popular throughout all cultures and ages in the modern world, and the fashion trend of denim is being changed continuously by a change in washing technology. The study shows the chronological change in denim washing technology. Therefore, this paper will help factories to forecast the future fashion of denim in the global market as well as the cost comparison among various washing technology.

\section{Methodology}

Bangladesh Denim Production market is facilitated with a large number of factories to support the demand over the world. Since denim washing is a very compulsory requirement for denim products, different denim washing plants are growing very rapidly. In this paper, all the data are primarily collected from washing factories. The washing process details and the costing was collected. The data was collected for the last nine years, beginning from 2010 to 2018. Following the data, the washing trend of the denim products was found out and plotted in this paper. This research is an exploratory research in nature which starts with the trend of denim wash technologies. Gradually, this study will aim at forecasting future denim wash trend. This will analyze the costing and their root cause of variation throughout the years. All the data are primary data and were collected directly based on direct observations.

\section{Sampling Design}

The population for this study includes all the denim washing industry in Bangladesh. For sampling purpose, ten denim washing factories are selected regarding their contribution to the market, environmental concerns, quality, and industrial popularity over the country. Thus, the sample size of 
this study is 10 . The data collected from each factory base on the washing techniques in which they operate. The average value was considered and the result was analyzed for each of the techniques. The sample selection was done due to a concern from Bangladesh Textile Mills Corporation (BTMC).

\section{Result and Discussion}

Various types of dry and wet washing techniques are being incorporated for the denim products. Moreover, the day by day change in the techniques is according to the buyer's requirement. This is because some techniques has been approached newly, some have faded away, some have been banned due to safety or environment concerns, etc. The following tables (Table 1 and 2) show the trend of different washing techniques from 2010 to 2018. However, from the table, it was found that in 2010, there was an extensive use of different common wet washing techniques such as desizing, enzyme wash, bleaching or softener treatment. Nevertheless, there are no more in extensive use as at present because they have been replaced with various dry processes gradually. Thus, from around 2013, different dry wash techniques were introduced into the market to facilitate the products with better quality at the shortest possible time and cost. Before 2013, there was a little practice of whiskering and scrapping to make a fade look pattern on denim garments. Currently, the dry techniques like tagging, destroy, crinkle, grinding, and PP Spray (potassium permanganate spray) are very popular in the denim market. On the other hand, from 2013, dry wash technique like sand blasting has been banned due to its harmful effects on operators and users.

Table 1. Trend of Dry Washing Techniques

\begin{tabular}{|c|c|c|c|c|c|c|c|c|c|c|}
\hline Yea & & 2010 & 2011 & 2012 & 2013 & 2014 & 2015 & 2016 & 2017 & 2018 \\
\hline \multirow{10}{*}{ 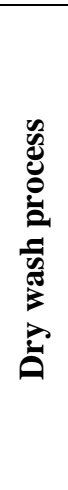 } & Whiskering & $\sqrt{ }$ & $\sqrt{ }$ & $\sqrt{ }$ & $\sqrt{ }$ & $\sqrt{ }$ & $\sqrt{ }$ & $\sqrt{ }$ & $\sqrt{ }$ & $\sqrt{ }$ \\
\hline & Scrapping & $\sqrt{ }$ & $\sqrt{ }$ & $\sqrt{ }$ & $\sqrt{ }$ & $\sqrt{ }$ & $\sqrt{ }$ & $\sqrt{ }$ & $\sqrt{ }$ & $\sqrt{ }$ \\
\hline & Tagging & $\sqrt{ }$ & $\sqrt{ }$ & $\sqrt{ }$ & $\sqrt{ }$ & $\sqrt{ }$ & $\sqrt{ }$ & $\sqrt{ }$ & $\sqrt{ }$ & $\sqrt{ }$ \\
\hline & Destroy & & & & & & $\sqrt{ }$ & $\sqrt{ }$ & $\sqrt{ }$ & $\sqrt{ }$ \\
\hline & Crinkle & & & & & & $\sqrt{ }$ & $\sqrt{ }$ & $\sqrt{ }$ & $\sqrt{ }$ \\
\hline & PP Spray & & & & $\sqrt{ }$ & $\sqrt{ }$ & $\sqrt{ }$ & $\sqrt{ }$ & $\sqrt{ }$ & $\sqrt{ }$ \\
\hline & Laser Whisker & & & & & & $\sqrt{ }$ & $\sqrt{ }$ & $\sqrt{ }$ & $\sqrt{ }$ \\
\hline & Laser Destroy & & & & & & $\sqrt{ }$ & $\sqrt{ }$ & $\sqrt{ }$ & $\sqrt{ }$ \\
\hline & $\begin{array}{l}\text { Full body } \\
\text { laser print }\end{array}$ & & & & & & & & & $\sqrt{ }$ \\
\hline & Sand Blasting & $\sqrt{ }$ & $\sqrt{ }$ & $\sqrt{ }$ & & & & & & \\
\hline
\end{tabular}


Table 2. Trend of Wet Washing Techniques

\begin{tabular}{|c|c|c|c|c|c|c|c|c|c|c|}
\hline Year & & 2010 & 2011 & 2012 & 2013 & 2014 & 2015 & 2016 & 2017 & 2018 \\
\hline \multirow{14}{*}{ 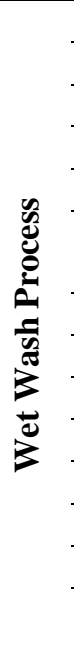 } & Desizing & $\sqrt{ }$ & $\sqrt{ }$ & $\sqrt{ }$ & $\sqrt{ }$ & $\sqrt{ }$ & $\sqrt{ }$ & $\sqrt{ }$ & $\sqrt{ }$ & $\sqrt{ }$ \\
\hline & Enzyme wash & $\sqrt{ }$ & $\sqrt{ }$ & $\sqrt{ }$ & $\sqrt{ }$ & $\sqrt{ }$ & & & & \\
\hline & Softener & $\sqrt{ }$ & $\sqrt{ }$ & $\sqrt{ }$ & $\sqrt{ }$ & $\sqrt{ }$ & $\sqrt{ }$ & $\sqrt{ }$ & $\sqrt{ }$ & $\sqrt{ }$ \\
\hline & Bleach & $\sqrt{ }$ & $\sqrt{ }$ & $\sqrt{ }$ & $\sqrt{ }$ & $\sqrt{ }$ & & & & \\
\hline & Acid wash & & & & & & $\sqrt{ }$ & $\sqrt{ }$ & $\sqrt{ }$ & $\sqrt{ }$ \\
\hline & $\begin{array}{l}\text { Enzyme stone } \\
\text { bleach wash }\end{array}$ & & & & & & $\sqrt{ }$ & $\sqrt{ }$ & $\sqrt{ }$ & $\sqrt{ }$ \\
\hline & Sand Blasting & $\sqrt{ }$ & $\sqrt{ }$ & $\sqrt{ }$ & & & & & & \\
\hline & Ozone wash & & & & & & & & & $\sqrt{ }$ \\
\hline & Towel Wash & & & & & & $\sqrt{ }$ & $\sqrt{ }$ & $\sqrt{ }$ & $\sqrt{ }$ \\
\hline & Full body laser print & & & & & & & & & $\sqrt{ }$ \\
\hline & Semi Bleach & & & & & & & & & $\sqrt{ }$ \\
\hline & Acid Enzyme Wash & & & & & & & $\sqrt{ }$ & $\sqrt{ }$ & $\sqrt{ }$ \\
\hline & Optical Wash & & & & & & & $\sqrt{ }$ & & \\
\hline & $\begin{array}{l}\text { Clean cup with } \\
\text { Brightener }\end{array}$ & & & & & & & & & $\sqrt{ }$ \\
\hline
\end{tabular}

Normal and common washing techniques like enzyme wash, bleaching, desizing or softener treatment were very popular since there was a little bit concern of various appearances from a denim garment. However, these techniques have gradually lost their appeal due to the inducement of high-performance washing techniques like acid wash, enzyme stone wash, and enzyme stone bleach wash. The wet washing market has been incorporated from around 2014-2015, having a supportive action with dry techniques. Some wet washing techniques are very recent in the market and are expected to be improved very soon due to their functionality and efficiency. Consequently, wet washing techniques like ozone wash, semi-bleach, clean cup with brightener, full body laser print, and acid enzyme wash are very common.

Laser destroy and laser whiskering are two very immersing techniques in the dry wash from near 2015 and are very extensive in use. This is because of their fast action and environment-friendly behavior despite the fact that initial investment is high. Thus, the Bangladesh dry washing techniques have a great approach as well as the newly approached wet processes for various denim garments.

The costing behavior in denim garments washing is rather simple and, with time, the costs have grown up for more or less in all the major washing techniques (dry and wet). Table 2 and 3 has shown the costing of different washing techniques and their changing pattern over the years from 2010 to 2018. In addition, the data have been plotted graphically on Figure 1 and 2 respectively. 
Table 3. Costing of different dry washing techniques

\begin{tabular}{llllll}
\hline \multirow{2}{*}{ Dry washing techniques } & \multicolumn{2}{l}{ Cost per piece(USD) } \\
\cline { 2 - 6 } & 2010 & 2012 & 2014 & 2016 & 2018 \\
\hline Whiskering & $\$ 0.25$ & $\$ 0.25$ & $\$ 0.30$ & $\$ 0.40$ & $\$ 0.40$ \\
\hline Scraping & $\$ 0.20$ & $\$ 0.20$ & $\$ 0.30$ & $\$ 0.30$ & $\$ 0.30$ \\
\hline Tagging & $\$ 0.10$ & $\$ 0.10$ & $\$ 0.15$ & $\$ 0.15$ & $\$ 0.15$ \\
\hline Destroy & $\$ 0.35$ & $\$ 0.35$ & $\$ 0.40$ & $\$ 0.50$ & $\$ 0.50$ \\
\hline Crinkle & $\$ 0.50$ & $\$ 0.50$ & $\$ 0.50$ & $\$ 0.60$ & $\$ 0.60$ \\
\hline Pocket edge and Hem grinding & $\$ 0.10$ & $\$ 0.10$ & $\$ 0.15$ & $\$ 0.20$ & $\$ 0.20$ \\
\hline PP Spray & $\$ 0.25$ & $\$ 0.25$ & $\$ 0.30$ & $\$ 0.30$ & $\$ 0.35$ \\
\hline Laser Whisker & $\$ 0.70$ & $\$ 0.80$ & $\$ 0.90$ & $\$ 1.00$ & $\$ 1.00$ \\
\hline Laser Destroy & $\$ 0.90$ & $\$ 1.00$ & $\$ 1.00$ & $\$ 1.20$ & $\$ 1.20$ \\
\hline
\end{tabular}

Table 4. Costing of different wet washing techniques

\begin{tabular}{llllll}
\hline \multirow{2}{*}{ Wet washing techniques } & \multicolumn{5}{l}{ Cost per piece (USD) } \\
\cline { 2 - 6 } & 2010 & 2012 & 2014 & 2016 & 2018 \\
\hline Enzyme wash & $\$ 0.25$ & $\$ 0.25$ & $\$ 0.20$ & $\$ 0.15$ & $\$ 0.15$ \\
\hline Enzyme stone wash & $\$ 0.75$ & $\$ 0.75$ & $\$ 0.80$ & $\$ 0.80$ & $\$ 0.85$ \\
\hline Acid wash & $\$ 0.70$ & $\$ 0.70$ & $\$ 0.80$ & $\$ 1.00$ & $\$ 1.00$ \\
\hline Enzyme stone bleach wash & $\$ 0.80$ & $\$ 0.80$ & $\$ 0.90$ & $\$ 0.90$ & $\$ 0.95$ \\
\hline Stone wash & $\$ 0.40$ & $\$ 0.45$ & $\$ 0.45$ & $\$ 0.50$ & $\$ 0.50$ \\
\hline Tinting & $\$ 0.09$ & $\$ 0.09$ & $\$ 0.10$ & $\$ 0.10$ & $\$ 0.10$ \\
\hline Ozone wash (3min) & $\$ 0.15$ & $\$ 0.15$ & $\$ 0.20$ & $\$ 0.20$ & $\$ 0.20$ \\
\hline Ozone wash (30min) & $\$ 0.45$ & $\$ 0.45$ & $\$ 0.50$ & $\$ 0.60$ & $\$ 0.60$ \\
\hline Towel wash & $\$ 0.90$ & $\$ 0.90$ & $\$ 1.00$ & $\$ 1.10$ & $\$ 1.10$ \\
\hline
\end{tabular}

$\$ 1.40$

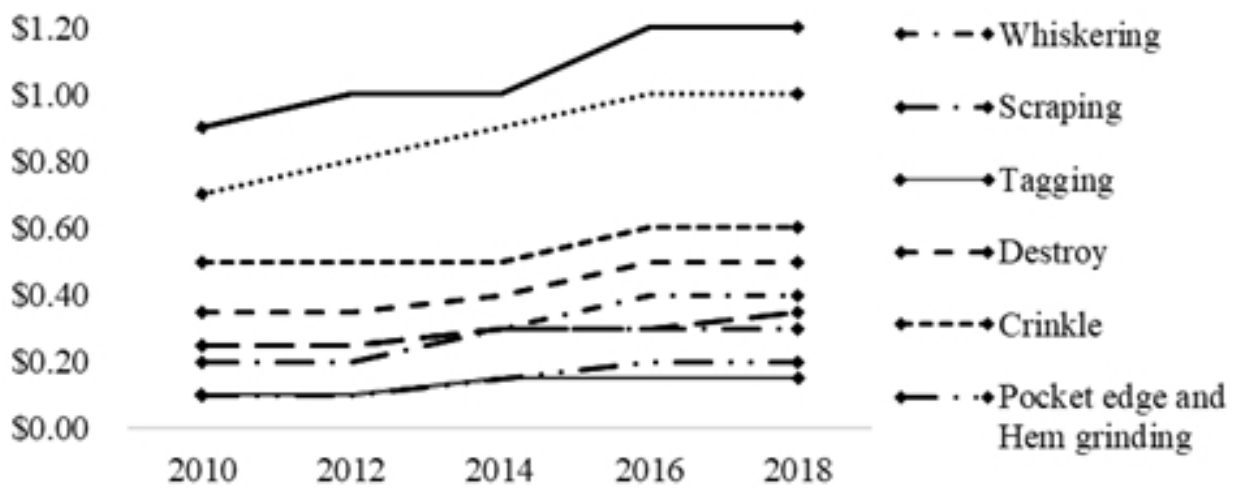

Figure 1. Costing of different dry wash techniques 


\section{$\$ 1.20$}
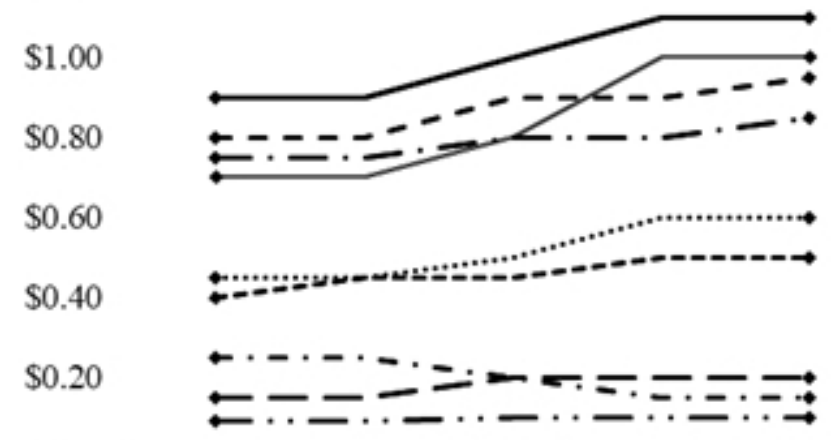

$\$ 0.00$ $\bullet \cdot-\bullet$ Enzyme wash

$\leftarrow \cdot \rightarrow$ Enzyme stone wash

-Acid wash

$-\rightarrow$ Enzyme stone

bleach wash

$\rightarrow$ Stone wash

$\leftarrow \cdots$ Tinting

$\leftarrow \rightarrow$ Ozone wash

(3min)

Ozone wash

(30min)

Figure 2. Costing of different wet wash techniques

From the graphical representation, it was found clearly that the costing of the majority of the techniques has been increasing gradually. This is basically due to the growing labor cost and material cost. However, the cost for enzyme wash has been decreased for the changed price of the enzyme in the market. Hence, it is predicted that quality denim washed products cost higher compared to the subsequent previous years. The costing of acid wash led to a greater change in cost over the years due to its various applications to provide the products with distinct appearances. The same situation is also applicable for laser whiskering effects. This is because laser treatment provides very sharp and distinct effect on the product. Also, the demand of the wash is very high in the market. However, the findings can be seen from the graphical presentations. After 2016, the costing of different wash techniques started remaining constant; thus, the costing is getting stable with the market. From this discussion, we can have a prediction about the costing that it may remain stable for the coming years except due to any unavoidable change.

\section{Conclusion}

This paper may assist to predict the future trend of denim washing in the global market as well as the costing behavior. To start a new washing plant or existing factories, these data can be analyzed to prepare their production planning and costing. This paper also helps to understand the current fashion of the denim world. The costing of denim washing varies due to different reason such as raw material availability, the quantity of order, and customers demand. The price included in this paper is average values and may slightly vary. The data used in this paper are collected from ten factories of different 
buyers or different brands. Consequently, the data represents the average value for all the factories used in this study, and it is not exact for any particular factory. However, the chronological change in costing represents the washing trend and its forecasting.

\section{References:}

1. HAQUE, M. S. (n.d.). PROCESSINGAND CHARACTERIZATIONOF W WASTE DENIM FIBER REINFORCED EINFORCED POLYMER COMPOSITES.

2. Historian, L. S. \& C. (2014). A SHORT HISTORY OF DENIM. Lynn Downey, 8(33), 44.

3. Iqbal, S., Guggenberger, M., \& Alam, K. (2012). Deadly Denim: Sandblasting in the Bangladesh Garment Industry, 52.

4. Island, R. (2014). BANGLADESH denim industry, (1), 32-34.

5. JCR-VIS. (2018). Denim Industry, 2016. Retrieved from http://jcrvis.com.pk/docs/Denim201803.pdf

6. Khan, M. M. R., Mondal, M. I. H., \& Uddin, M. Z. (2013). Sustainable washing for denim garments by enzymatic treatment. Journal of Chemical Engineering, 27(1), 27-31. https://doi.org/10.3329/jce.v27i1.15854

7. Khan, M. R., \& Mondal, I. H. (2018). Sustainable Washing for Denim Garments by Enzymatic Treatment Sustainable Washing for Denim Garments by Enzymatic Treatment, C(January), 27-31. https://doi.org/10.3329/jce.v27i1.15854

8. Profile, S. E. E. (2014). Evaluation of Mechanical Properties of Denim Garments after Enzymatic Bio- Washing, (January). https://doi.org/10.5829/idosi.wasj.2014.31.09.118

9. Trends, G., The, I. N., Sector, G., \& For, O. (2016). GLOBAL TRENDS IN THE GARMENT SECTOR AND Readymade Garment $\&$ Textile Industry in Bangladesh. 